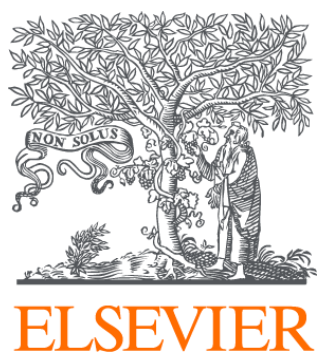

Since January 2020 Elsevier has created a COVID-19 resource centre with free information in English and Mandarin on the novel coronavirus COVID-

19. The COVID-19 resource centre is hosted on Elsevier Connect, the company's public news and information website.

Elsevier hereby grants permission to make all its COVID-19-related research that is available on the COVID-19 resource centre - including this research content - immediately available in PubMed Central and other publicly funded repositories, such as the WHO COVID database with rights for unrestricted research re-use and analyses in any form or by any means with acknowledgement of the original source. These permissions are granted for free by Elsevier for as long as the COVID-19 resource centre remains active. 


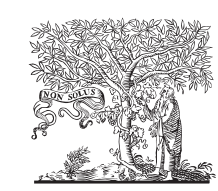

ELSEVIER

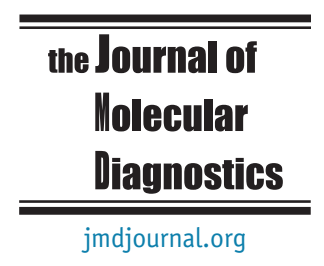

\title{
Two-Stage Hierarchical Group Testing Strategy to Increase SARS-CoV-2 Testing Capacity at an Institution of Higher Education
}

\section{A Retrospective Analysis}

\author{
Troy J. Ganz, * Rachel Donner, * Kevin M. Hines, * Markus L. Waithe-Alleyne, * Deirdre L. Slate, * Gyorgy Abel, *† and \\ Jared R. Auclair*
}

From the Department of Chemistry and Chemical Biology, * Life Sciences Testing Center, Northeastern University Innovation Campus in Burlington, Burlington, Massachusetts; and Lahey Hospital and Medical Center, ${ }^{\dagger}$ Burlington, Massachusetts

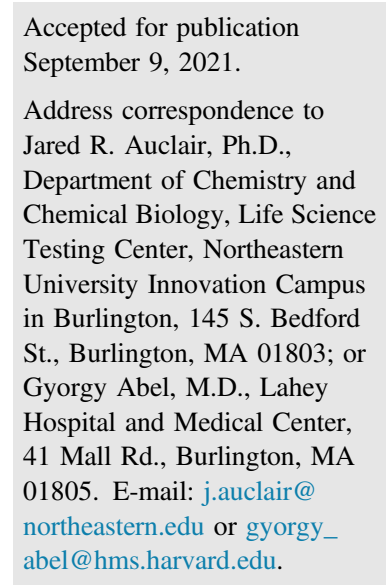

\begin{abstract}
Population testing for severe acute respiratory syndrome coronavirus 2 is necessary because of the potential for viral transmission from asymptomatic cases, yet the scarcity of reagents and equipment has increased the cost-prohibitive implementation of screening campaigns at institutions of higher education. Significant analytical sensitivities of nucleic acid amplification methods permit sample pooling to increase testing capacity. Statistical models compared optimal testing configurations for pools of 3, 5, and 10 samples. Assessment of pooling using the TaqPath COVID-19 Combo Kit multiplex assay (ORF1ab, $N$, and $S$ gene targets) involved a limit-of-detection study, matrix-effect study, and clinical comparison of neat with pooled samples. A limit of detection of 135.02 (ORF1ab; 95\% CI, 117.21-155.52), 373.92 ( $N$; 95\% CI, 257.05-437.64), and 1001.32 (S; 95\% CI, 896.62-1118.33) gene copy equivalents per milliliter was resolved. Seventy-two randomly selected samples showed slight suppression owing to a negative sample matrix. The resulting mean cycle threshold shifts were 2.09 $($ ORF1ab), $1.76(N)$, and $2.31(S)$ for the 3-sample pool, $2.83($ ORF1ab), $2.45(N)$, and $3.24(S)$ for the 5-sample pool, and $3.99($ ORF1ab), $3.46(N)$, and $4.07(S)$ for the 10-sample pool. Despite a quantitative sensitivity loss trend, the qualitative result was unaffected in each pool. According to the range of disease prevalence observed at the testing site $(0.03 \%$ to $7.32 \%)$, a pool of five samples was deemed an optimal and cost-effective option for monitoring the Northeastern University community. (J Mol Diagn 2021, 23: 1691-1698; https://doi.org/10.1016/j.jmoldx.2021.09.001)
\end{abstract}

The emerging pandemic of coronavirus disease-2019 (COVID-19), sourced from the novel strain of $\beta$-coronavirus known as severe acute respiratory syndrome coronavirus 2 (SARS-CoV-2), has had a severe impact on global health care systems. ${ }^{1}$ Institutions of higher education have been confronted with the conflicting obligations to guarantee a standard quality of education while ensuring the safety of their faculty, staff, and students during the pandemic. For universities with shared living, dining, transportation, and classroom learning arrangements, the greater requirements of risk management must be weighed against the economic loss of closing for an indeterminate period. ${ }^{2}$ Further pressures are imposed on institutions of higher education in urban environments as positive correlations of transmission have been observed with factors such as reduced air quality and higher population density. ${ }^{3}$ Similar to other respiratory infections, transmission is facilitated by viral shedding in the upper respiratory tract, which may release viral particles in the form of aerosols $(\leq 5 \mu \mathrm{m})$ or droplets $(>5 \mu \mathrm{m})$ to contaminate the surrounding air or surfaces, and spread via fomites, coughing, sneezing, and exhalation from symptomatic, as well as presymptomatic and asymptomatic,

Disclosures: None declared. 
carriers. ${ }^{4,5}$ Along with recommended measures for risk management such as physical distancing, obligatory personal hygiene, and mask wearing, a rigorous screening and surveillance campaign provides the most effective response.

Quantitative reverse transcription-polymerase chain reaction (RT-qPCR) offers the most sensitive method for early detection of SARS-CoV-2 when compared with other available analytical methodologies, which may require larger viral loads or long periods of seroconversion after infection. ${ }^{6}$ In addition, accessibility of less-invasive nasopharyngeal, oropharyngeal, and nare respiratory swab collections have demonstrable stability and result in better patient compliance. ${ }^{7}$ Drawbacks coincide with the sharp increase in global demand for medical and testing supplies such as personal protective equipment (gloves, gowns, and surgical and N95 masks), collection swabs and containers, transport and lysis buffers, nucleic acid extraction and amplification kits, consumables (eg, micropipette tips), analytical equipment, and qualified technical personnel because the high-complexity format of RT-qPCR testing schema requires a reliable supply chain of critical materials.

Group testing, or sample pooling, is an attractive method to increase testing capacity without the need for additional resources or training. Methods in statistical modeling have been developed to predict optimal group sizes for both hierarchical and nonhierarchical testing algorithms parameterized by disease prevalence and testing error. ${ }^{8}$ Pressured by the COVID-19 pandemic, investigators have sought to validate SARS-CoV-2 group testing on available molecular platforms. ${ }^{9-11}$ Because persistent evolution of SARS-CoV2 variants pose a threat to vaccine efficacy, Northeastern University expects population screening to extend until evidence of a significant reduction in viral reproductive rate is attained. Thus, the following study sought to define an ideal pooling size with consideration of the impact on analytical sensitivity and operations efficiency because implementation of group testing will be crucial for an economical continuation of COVID-19 response.

\section{Materials and Methods}

\section{Study Demographics, Sample Collection, and Pre- Analytical Processing}

This study was conducted at the Life Sciences Testing Center (LSTC; Northeastern University, Burlington, MA) for Northeastern University students, faculty, and staff at regional campuses primarily in Boston, MA; Portland, ME; and Charlotte, NC. Since the establishment of the LSTC in August 2020, the site has observed an overall disease prevalence of $0.22 \%$ (95\% CI, $0.11 \%-0.33 \%$ ). Students attending campus were required to test every 3 days $( \pm 1$ day), and employees on site 5 days a week were expected to test at least twice a week. Because all affiliates are directed to a designated symptomatic or asymptomatic collection site based on their claims to have had COVID-19 symptoms or close contact with a COVID-19-positive case within the previous 24 hours, the LSTC maintained a curated repository comprising more than 1800 positive samples at a ratio of 3.3:1 asymptomatic to symptomatic cases. Relative prevalence rates were $3.86 \%$ (95\% CI, $2.37 \%-5.34 \%$ ) for symptomatic and $0.17 \%(95 \% \mathrm{CI}, 0.08 \%-0.25 \%)$ for asymptomatic cohorts.

Anterior nare polyester swabs were collected (15 seconds per nostril) in 3-mL BD Vacutainer (cat: 366703; without additives, Mississauga, ON, Canada) tubes and transported dry at ambient temperature. Dry swab stability was fewer than 24 hours at ambient temperature and samples were shipped via local courier or priority overnight. Processing involved the addition of $3 \mathrm{~mL}$ viral transport medium (cat: VR2019-1L; Redoxica, Little Rock, AR) and shaking at $1 \mathrm{x}$ $\mathrm{g}$ for 5 minutes. Rehydrated specimens were stable for fewer than 72 hours at $2^{\circ} \mathrm{C}$ to $8^{\circ} \mathrm{C}$, and indefinitely at $-80^{\circ} \mathrm{C}$.

\section{Ethics Statement}

Retrospective samples were deidentified for study purposes in accordance with Institutional Review Board exemption by the Northeastern University Office of Human Subject Research Protection.

\section{Statistical Modeling}

The prospective workflow was modeled using an adaptive two-stage hierarchical algorithm, whereby results from the master pool require testing of the individual samples. An optimal testing configuration was the result of parameters including disease prevalence, group size, analytical sensitivity, and analytical specificity. Under nonpooling conditions, total assays dependent on the assay controls could be expressed as follows: $E=n+1$, where $\mathrm{E}$ is the expected number of tests and $\mathrm{n}$ is the probability of repeat testing resulting from an invalidated test (eg, internal extraction control failure, external quality control failure). Pursuant to LSTC protocol, an invalid test was repeated once and further representation for the total assays were expressed as follows: $E=\left(n^{2}\right)+(n+1)$. Using an open-source statistical modeling application (University of Nebraska-Lincoln, www.chrisbilder.com/shiny, last accessed September 1, 2020), the term $E$ was included to achieve estimated reductions for pools of 3, 5, and 10 samples. Sensitivity was set to $95 \%$ based on the calculated limit of detection, while specificity was set to 99\% under the combined influence of the assay controls and operator review.

\section{Analytical Processing: RNA Extraction and RT-qPCR}

SARS-CoV-2 RNA was extracted from a $200-\mu \mathrm{L}$ sample using the Applied Biosystems MagMAX Viral/Pathogen Nucleic Acid Isolation Kit (cat: A48383; Thermo Fisher Scientific, Waltham, MA) on semi-automated Agilent Bravo 
liquid handlers (Agilent Technologies, Santa Clara, CA). Briefly, each well in a 1-mL, 96-well plate was prepared with $5 \mu \mathrm{L}$ proteinase $\mathrm{K}, 200 \mu \mathrm{L}$ sample, $275 \mu \mathrm{L}$ lysis buffer/ binding beads, and $5 \mu \mathrm{L}$ bacteriophage MS2 control. It was then shaken for 2 minutes at $3 \mathrm{x} \mathrm{g}$ and incubated for 5 minutes at $65^{\circ} \mathrm{C}$. Incubation on a magnetic plate at room temperature allowed aspiration of waste material and a sample undergoes three cycles of resuspension/aspiration in $165 \mu \mathrm{L}$ wash buffer, $165 \mu \mathrm{L} 80 \%$ ethanol, and $50 \mu \mathrm{L}$ elution buffer, respectively. After a final separation event, $50 \mu \mathrm{L}$ purified RNA solution was transferred to a fresh 1-mL, 96well plate.

RT-qPCR was performed according to the US Food and Drug Administration emergency use authorization instructions for use for the Applied Biosystems TaqPath COVID-19 Combo kit (cat: A47814; Thermo Fisher Scientific, Waltham, MA), which is a multiplex assay to detect SARS-CoV-2 ORFlab, $N$, and $S$ genes, in addition to a spiked-in MS2 bacteriophage extraction control (reporter dyes $\mathrm{FAM}^{\mathrm{TM}}, \mathrm{VIC}^{\circledR}, \mathrm{ABY}^{\circledR}$, and $\mathrm{JUN}^{\circledR}$, respectively). A Micro-Amp Optical 96-well reaction plate (cat: 4326659, ThermoFisher, Waltham, MA) was prepared with $10 \mu \mathrm{L}$ purified RNA and $15 \mu \mathrm{L}$ reaction mix, which includes TaqPath 1-Step Multiplex Master Mix (cat: A28523; No ROX, Thermo Fisher, Waltham, MA), primers, and probes. Thermal profile parameters involved a 2 -minute uracil-Nglycosylase (UNG) incubation cycle at $25^{\circ} \mathrm{C}$, a 10 -minute reverse transcriptase incubation cycle at $53^{\circ} \mathrm{C}$, a 2-minute activation cycle at $95^{\circ} \mathrm{C}$, followed by 40 cycles of a 3second denaturation at $95^{\circ} \mathrm{C}$, and a 30-second anneal/ extension cycle at $60^{\circ} \mathrm{C}$ on an Applied Biosystems 7500 Fast Dx Real-Time PCR Instrument (cat: 4406985; Thermo Fisher Scientific, Waltham, MA). Threshold cycle $\left(\mathrm{C}_{\mathrm{T}}\right)$ values were exported using Applied Biosystems COVID-19 Interpretive Software (version 1.5), which determined a valid extraction for MS2 $\left(C_{T} \leq 32\right)$ and qualitative SARSCoV-2 positive if two of three genes are detected at $\mathrm{C}_{\mathrm{T}} \leq$ 37 , inconclusive if one of three genes is detected at $C_{T} \leq 37$, or negative for genes detected at $C_{T}>37$. External controls (positive and negative) were included with every run.

\section{Analytical Sensitivity in Sample Matrix}

A limit of detection (LOD) study was designed using pooled negative sample material spiked with SARS-CoV-2 viral control (ATCC VR-1986HK; strain: 2019-nCoV/USA-WAl/ 2020; Manassas, VA). Serial dilutions were performed in two extraction plates on different days. The first series included concentrations of $3.9 \times 10^{6}, 1.95 \times 10^{6}, 487,500$, $122,000,30,500,7600,2000,500,200,30$, and 10 gene copy equivalent (gce)/mL, and the second series included concentrations of 39,000, 19,500, 3900, 2000, 1000, 500, 200, 100, 30, and $10 \mathrm{gce} / \mathrm{mL}$; negative sample pools were additionally assayed separately to rule out the presence of interfering substances. Each concentration was extracted from eight replicates and RT-qPCR was performed in triplicate for each plate. The resulting $\mathrm{C}_{\mathrm{T}}$ values were compiled in Microsoft Excel (Redmond, WA) whereby success rates were calculated from qualifying $\mathrm{C}_{\mathrm{T}}$ values $(\leq 37)$ over 24 trials.

\section{Matrix Effect and Dilution Comparison Study}

Seventy-two positive samples with average $\mathrm{C}_{\mathrm{T}}$ values of 19.53 (ORF1ab; 95\% CI, 18.41-20.65), 20.17 ( ; 95\% CI, 19.11-21.22), and $19.18(S ; 95 \%$ CI, 17.48-20.89; $n=34$ ) were extracted undiluted (neat) and at dilutions of $3 \times, 5 \times$, and $10 \times$ using the negative sample pool as diluent in one series and viral transport medium as diluent in another series. Negative sample pools were constructed to simulate pool size; thus, pools of two, four, and nine negative samples accounted for $135 \mu \mathrm{L}, 160 \mu \mathrm{L}$, and 180 $\mu \mathrm{L}$ of the extraction volumes for pools 3,5 , and 10 , to which $65 \mu \mathrm{L}, 40 \mu \mathrm{L}$, and $20 \mu \mathrm{L}$ of positive sample were added, respectively. In addition, pools of three negative samples were generated to study the performance of two positive samples in pools of five. As a follow-up evaluation, 14 weak positive samples with average $C_{\mathrm{T}}$ values of 27.36 (ORF1ab; 95\% CI, 26.35-28.38), 27.98 ( $N ; 95 \% \mathrm{CI}$, 27-28.96), and 28.21 ( $S$; 95\% CI, 26.66-29.75, $n=13$ ) were selected and analyzed separately in pools of five.

A matrix effect was calculated as the difference in $C_{T}$ value in the negative sample pool from standard (viral transport medium) at the relative dilution factor, which provided a result suggesting signal suppression (matrix effect, $<0$ ) or enhancement (matrix effect, $>0$ ). Similarly, a $\mathrm{C}_{\mathrm{T}}$ shift resulting from dilution was calculated by subtracting the neat result from the pooled result. Statistical analysis was performed using GraphPad Prism, version 9.0.0 (GraphPad Software, La Jolla, CA).

\section{Results}

\section{Statistical Modeling}

The average rate of invalid tests from December 2020 to June 2021 was $5.43 \%$ (95\% CI, 3.56\%-7.29\%), of which $0.46 \%$ (95\% CI, $0.26 \%-0.66 \%$ ) were owing to internal extraction control failure and the remainder was owing to external control failure, suspected contamination, or technical error. Consideration of these probabilities affecting a pooled sample twice in succession informed the testing reductions of 5000 samples for pools of 3, 5, and 10 samples (Figure 1). Over the range of repeat rates, the 10-sample pool was optimal at a prevalence of less than $2 \%$. However, the 5-sample pool consistently outperformed both the 3sample pool and 10-sample pool formats until prevalence was $>10 \%$. Interestingly, the impact of repeat testing owing to invalid assays appeared to be overtaken by deconvolution at increased disease prevalence. Examination within the range of expected prevalence $(0.03 \%$ to $7.32 \%)$ indicated 
$2.5 \%$ Reapeat Rate

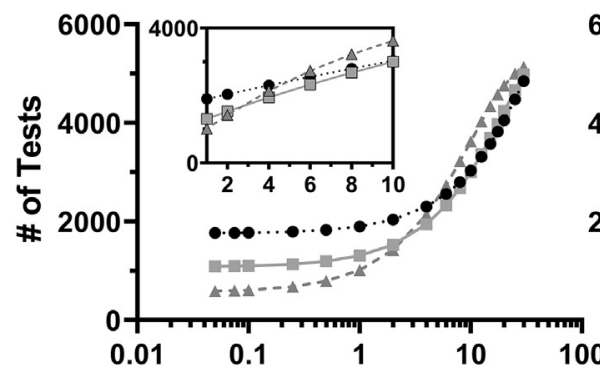

$5 \%$ Repeat Rate

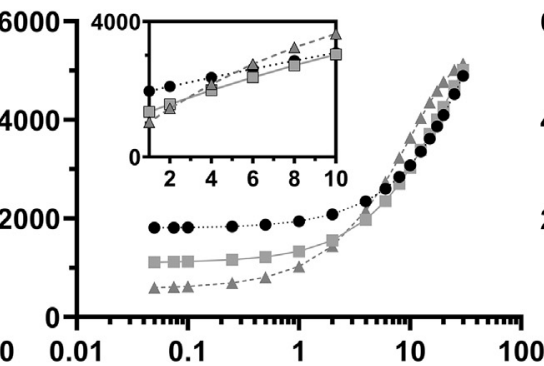

$10 \%$ Repeat Rate

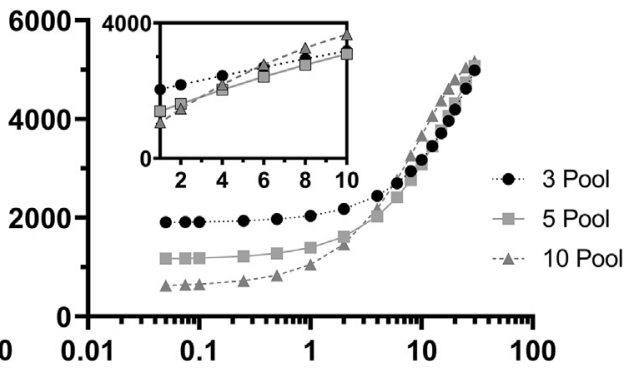

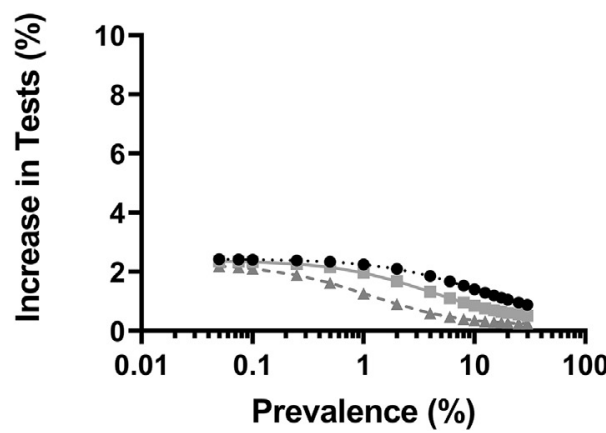
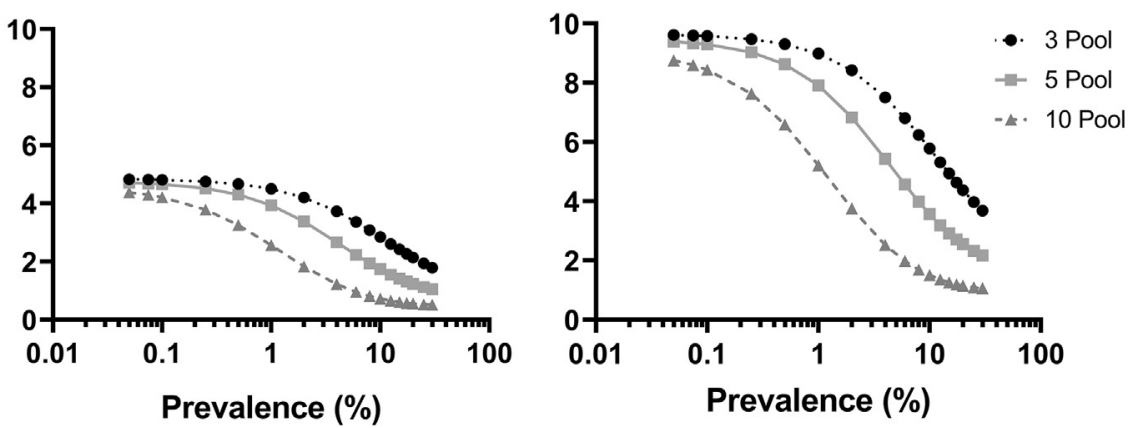

Figure 1 Statistical modeling. Top row: Expected number of tests for 5000 patients at repeated well (invalid) rates of $2.5 \%, 5 \%$, and $10 \%$ versus positive test prevalence. Insets: Prevalence range from $1 \%$ to $10 \%$. Bottom row: Increase (as percentage difference over a hypothetical $0 \%$ repeat rate) in testing by repeated well rates of $2.5 \%, 5 \%$, and $10 \%$ versus positive test prevalence. 3 -sample pool, black circles; 5 -sample pool, light gray squares; 10 -sample pool, dark gray triangles.

that the pooling method influences a favorable decrease in testing with increased pool size.

\section{Analytical Sensitivity in Sample Matrix}

Probit analysis was performed from combined runs to generate an LOD of $135.02 \mathrm{gce} / \mathrm{mL}$ (ORFlab; 95\% CI, $117.21-155.52 \mathrm{gce} / \mathrm{mL}), 373.92 \mathrm{gce} / \mathrm{mL}(N ; 95 \% \mathrm{CI}$, $257.05-437.64 \mathrm{gce} / \mathrm{mL})$, and $1001.32 \mathrm{gce} / \mathrm{mL}(S ; 95 \%$ CI, 896.62-1118.33 gce/mL) (Table 1). Consistent with the TaqPath COVID-19 Combo Kit instructions for use (Publication No: MAN0019181), which reported an LOD of 10 gce per reaction, a three of three genes result after the LOD for the $S$ gene was equivalent after a 10.01 gce/ reaction. Further application of the resulting algorithm for an inconclusive (one of three genes) and positive (two of three genes) result indicated estimated LODs of 200 gce/ $\mathrm{mL}$ (two gce/reaction) and $500 \mathrm{gce} / \mathrm{mL}$ (five gce/reaction), respectively. All negative pools were determined negative.

\section{Matrix Effect and Dilution Comparison Study}

Graphic analysis of the matrix effect suggested a central tendency toward a negligible impact of the sample matrix on pooled positive results because targets generally showed leptokurtic distributions centered proximal to zero (Figure 2). Accordingly, the 95\% CIs of the mean for each target of the 3-sample pool (ORFlab: -0.23 to $0.21 ; N$ : $0.05-0.25$; and $S:-0.59$ to -0.01$)$, 5-sample pool

Table 1 LOD Results for the Thermo Fisher TaqPath COVID-19 Combo Kit

\begin{tabular}{lllllllll}
\hline & \multicolumn{6}{l}{ Number detected/total replicates at each dilution, gce/mL (\% positive rate) } & \\
\cline { 2 - 6 } Target & 2000 & 1000 & 500 & 200 & 100 & 30 & 10 & LOD, gce/mL (95\% CI) \\
\hline ORF1ab & $48 / 48(100 \%)$ & $24 / 24(100 \%)$ & $48 / 48(100 \%)$ & $47 / 48(98 \%)$ & $22 / 24(92 \%)$ & $22 / 48(46 \%)$ & $4 / 48(8.3 \%)$ & 135.02 \\
$N$ gene & $48 / 48(100 \%)$ & $24 / 24(100 \%)$ & $48 / 48(100 \%)$ & $42 / 48(88 \%)$ & $13 / 24(54 \%)$ & $7 / 48(15 \%)$ & $1 / 48(2.1 \%)$ & $\begin{array}{l}(117.21-155.52) \\
(25.92\end{array}$ \\
S gene & $48 / 48(100 \%)$ & $23 / 24(96 \%)$ & $33 / 48(69 \%)$ & $0 / 48(0 \%)$ & $1 / 24(4.2 \%)$ & $0 / 48(0 \%)$ & $0 / 48(0 \%)$ & 1001.32 \\
& & & & & & & $(896.62-1118.33)$ \\
\hline
\end{tabular}

SARS-CoV-2 targets the ORF1ab, $N$, and $S$ genes.

gce, gene copy equivalents; LOD, limit of detection. 

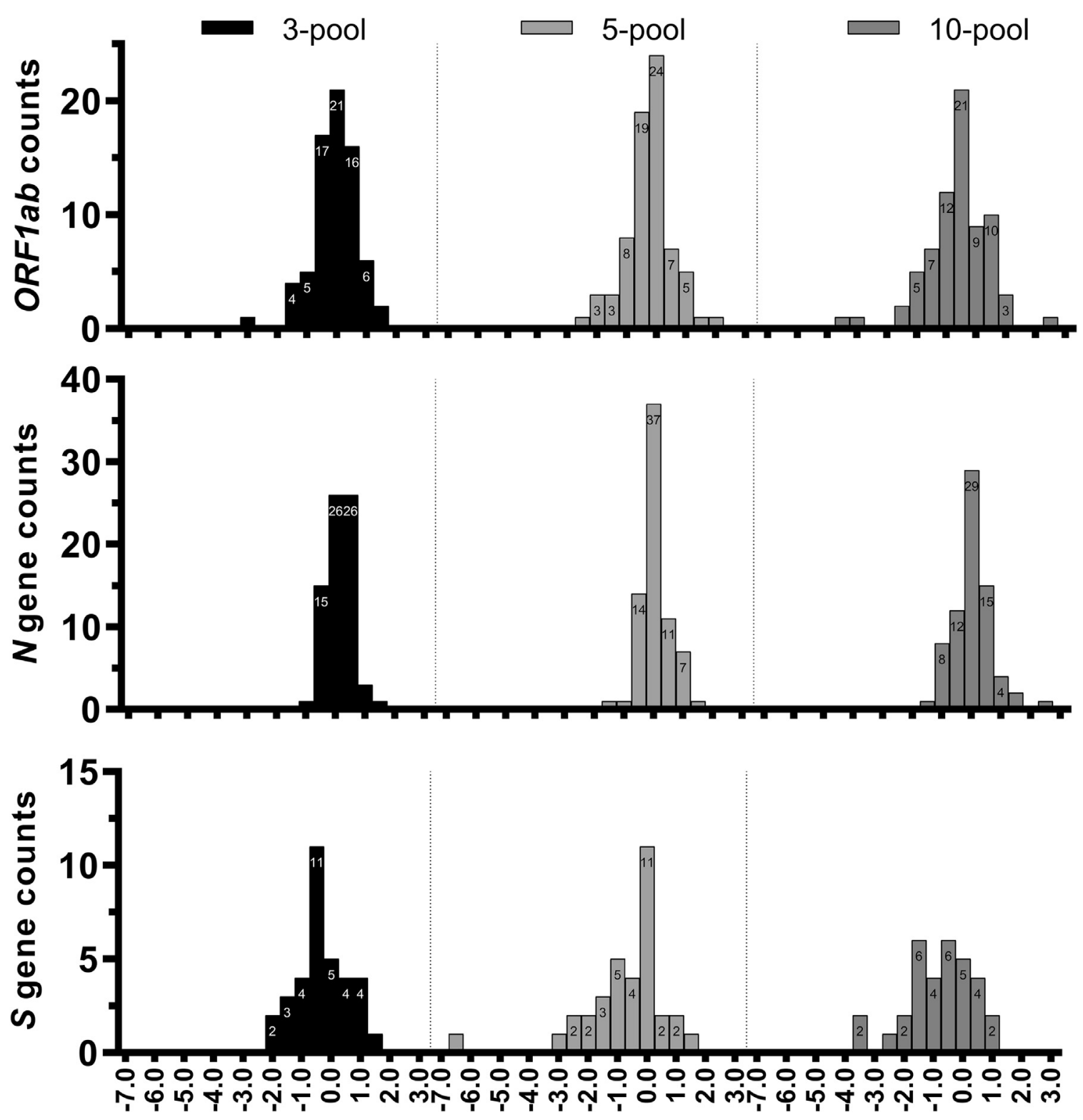

$\Delta \mathrm{Ct}$ value

Figure 2 Matrix-effect histogram analysis. Representative distributions for the threshold cycle $\left(C_{T}\right)$ shift observed for 72 positive samples produced by subtracting the result in the negative sample pool from the result in viral transport media. A positive $C_{T}$ shift indicates signal enhancement; a negative $C_{T}$ shift indicates signal suppression (bins $=0.5 C_{T}$ ).

(ORFlab: -0.45 to $-0.08 ; N$ : -0.03 to 0.18 , and $S:-1.22$ to -0.23 ), and 10-sample pool (ORF1ab: -0.86 to -0.36 ; $N$ : -0.11 to 0.19 ; and $S:-1.18$ to -0.38 ) supported an observation of slight suppression of less than one cycle for two of three genes.

Comparison of pooled with neat results provided average $\mathrm{C}_{\mathrm{T}}$ shifts of 2.09 (ORFlab; 95\% CI, 1.89-2.29), $1.76(\mathrm{~N}$; 95\% CI, 1.65-1.86), and $2.31(S ; 95 \% \mathrm{CI}, 1.98-2.64)$ for the 3-sample pool, 2.83 (ORFlab; 95\% CI, 2.61-3.05), $2.45(N ; 95 \% \mathrm{CI}, 2.34-2.55)$, and $3.24(S ; 95 \% \mathrm{CI}$, 2.74-3.73) for the 5-sample pool, and 3.99 (ORFlab; $95 \%$ CI, 3.76-4.22), $3.46(N$; 95\% CI, 3.33-3.58), and $4.07(S$; 95\% CI, 3.69-4.45) for the 10-sample pool. Despite an increase of less than 1 cycle for each gene between the 3and 5-sample pool, a paired $t$-test determined a statistically significant difference between the magnitude of $\mathrm{C}_{\mathrm{T}}$ shift $(P<0.001)$. Nevertheless, the Pearson $\mathrm{r}$ analysis reported excellent correlation between each pool with respect to the neat sample $\mathrm{C}_{\mathrm{T}}$ value $(r>0.97)$. Bland-Altman analysis, which describes the limits of agreement for two methods, offerred insight into the significance of the method comparison because each pooling strategy produced a CI of the bias (Figure 3) that did not include the line of equality $(y=0)$. Aside from a steady increase in bias with pooling, Bland-Altman plots illustrated tight grouping patterns within the $95 \%$ limits of agreement, which for the 3-sample 
ORF1ab
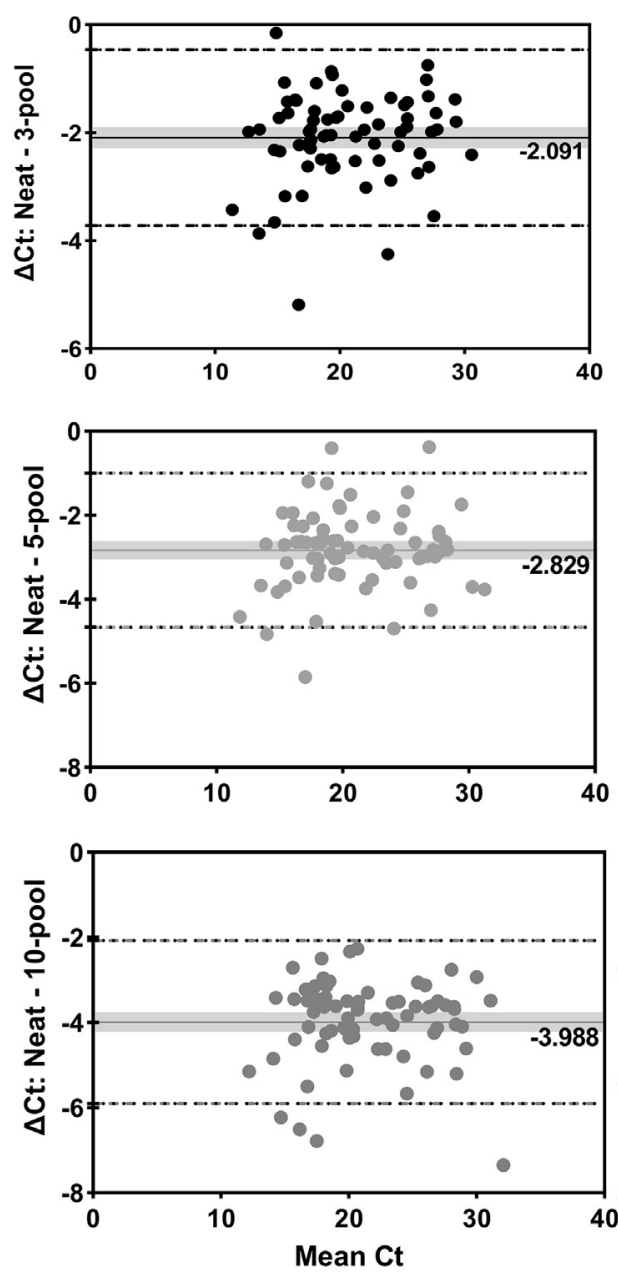

N gene
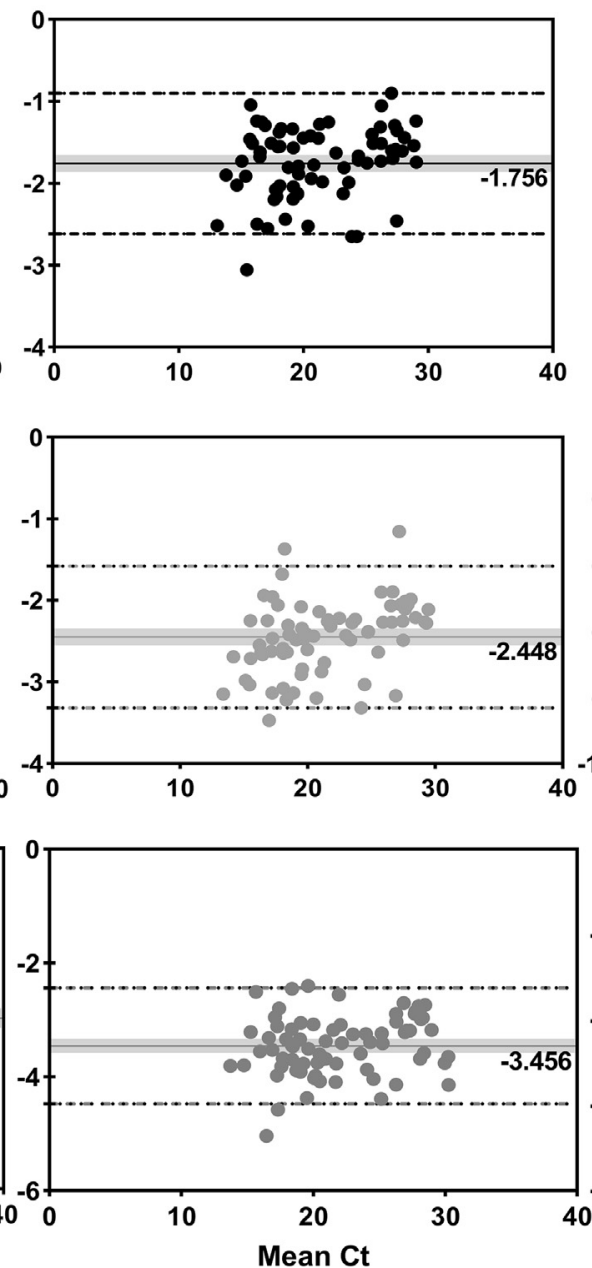

S gene
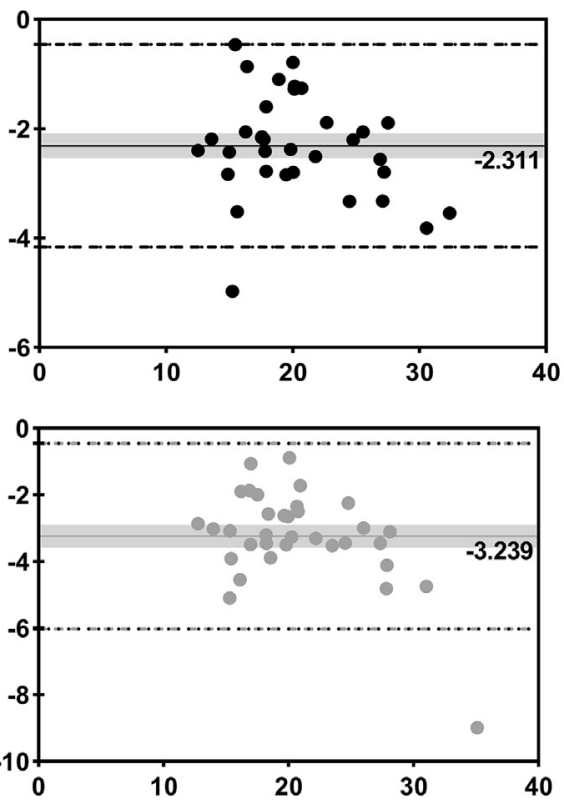

Figure 3 Bland-Altman (BA) analysis to show agreement between individual and pooling methods. BA plots constructed from the difference of the neat to pooled result as a function of the mean of the two results for each gene target at each pool size. Bias, solid line; $95 \%$ CI of the mean, shaded area; $95 \%$ limit of agreement, dashed lines; 3-sample pool, black circles; 5-sample pool, light gray circles; 10-sample pool, dark gray circles. $\mathrm{C}_{\mathrm{T}}$, threshold cycle.

pool targets were -3.72 to $-0.46($ ORFlab $),-2.62$ to $-0.89(N)$, and -4.17 to $-0.46(S)$; while the 5-sample pool targets were -4.67 to -0.99 (ORFlab), -3.32 to $-1.58(N)$, and -6.02 to $-0.46(S)$; and the 10 -sample pool targets were -5.91 to -2.7 (ORFlab),-4.47 to $-2.44(N)$, and -6.12 to $-2.02(S)$ (Figure 3).

Regarding the performance of a multiplex assay, $47.2 \%$ of the 72 samples were classified previously as showing $S$ gene target failure (SGTF) and determination was based on sensitivity to ORF $1 a b$ or $N$ gene targets. None of the targets were lost in the 3-sample pool dilution, only one sample lost detection of an $S$ gene in the 5-sample pool dilution, and two samples lost detection of an $S$ gene in the 10-sample pool dilution. Interestingly, a single sample determined an SGTF in both the neat and the 3-sample pool treatments was determined to have the $S$ gene in both the 5-sample pool and the 10 -sample pool conditions. All 72 samples showed absolute qualitative agreement under each pooling condition.
Further examination of 5-sample pools containing two positive samplesinvolved the same 72 samples permutated into 62 pairs. Because $48.4 \%$ were paired SGTF samples and only $12.9 \%$ were paired three-gene positives, analysis was performed on the ORFIab and $N$ genes. Bland-Altman analysis comparing the pooled result with the individual sample with the lowest $\mathrm{C}_{\mathrm{T}}$ indicated significant recovery of neat signal, reporting a bias of -0.227 (ORFlab; 95\% CI, -0.474 to 0.019$)$ and $-0.323(N ; 95 \% \mathrm{CI},-0.482$ to $-0.165)$, which concurred with the paired $t$-test for ORF1ab $(P>0.05)$ and $N$ genes $(P<0.001)$ (Supplemental Figure $\mathrm{S} 1)$.

A final set of 14 weak-positive samples tested in pools of five showed average $\mathrm{C}_{\mathrm{T}}$ shifts that were consistent with the previous set at 2.03 (ORFlab; 95\% CI, 1.25-2.81), $2.01(N ; 95 \% \mathrm{CI}, 1.33-2.68)$, and $3.12(S ; 95 \% \mathrm{CI}$, $0.78-5.45$ ), with only $23.1 \%$ (3 of 13) loss of $S$ gene detection owing to dilution. Nevertheless, the weak- 
positive sample set also showed absolute qualitative agreement (Supplemental Table S1).

\section{Discussion}

Sample pooling as an accessible method to increase testing capacity is dependent on a valid approach to mitigate altered target stability and sensitivity resulting from dilution in a variable matrix. Results from this study show that the relative stability to pool 3, 5, and 10 anterior nare swabs before RNA extraction for RT-qPCR analysis using the TaqPath COVID-19 Combo Kit as absolute qualitative agreement was retained under each pooled condition for the 86 samples selected. Combined analysis of statistical modeling paired with anticipated method error indicated optimal testing reductions and acceptable sensitivity loss for pools of five samples in the range of prevalence $(0.03 \%$ to $7.32 \%$ ) and viral loads shown by cohorts tested at the LSTC. Despite improved predicted reductions with pools of 10 at a prevalence between $0.03 \%$ and $2 \%$, temporaneous fluctuations in daily positivity rates combined with an estimated approximately four $\mathrm{C}_{\mathrm{T}}$ reduction in sensitivity suggests an introduction of unnecessary risk that was wellmitigated by pools of five. Furthermore, while pooling five samples may present favorable operational measures (eg, pipetting, sample tracking), reduction of the impact of control failures was improved over pools of three. Estimations are coincident with US Food and Drug Administration recommendations for pooling method validation version July 28, 2020 (US Food \& Drug Administration, https:// www.fda.gov/regulatory-information/search-fda-guidancedocuments/policy-coronavirus-disease-2019-tests-duringpublic-health-emergency-revised, last accessed June 21, 2021). ${ }^{12}$

Emerging SARS-CoV-2 variants show point mutations that are not only advantageous to strain proliferation via enhanced infectivity, transmissibility, and capacity to evade host immunity, but also confound target identification of RT-qPCR diagnostics. ${ }^{12}$ Variant of concern B.1.1.7 $(\alpha$ variant) harbors a six-nucleotide deletion in the spike gene, a mutation commonly referred to as $\Delta H 69 / \Delta V 70$, which has affected the TaqPath COVID-19 Combo Kit sensitivity toward the $S$ gene, causing SGTF. ${ }^{13}$ Of the 40 samples that showed SGTF, the ratio of asymptomatic to symptomatic cases of three:one was consistent with the case ratio of the LSTC-positive sample repository, thus a correlation between symptomology and proxy variant detection cannot be stated. Independent of SGTF, the $S$ gene showed the highest LOD, greatest variability in the negative sample matrix, and was the only target loss in pools of 5 and 10 . Therefore, prevalence of variants of concern may be irrelevant with consideration for the inherently improved positive predictive value of deconvolution methods that incidentally would refine proxy variant detection. Nevertheless, to supplement testing reduction, asymptomatic samples can be pooled while samples from symptomatic collection sites can continue to be tested individually.

The COVID-19 pandemic has disproportionately affected the mission of institutions of higher education with impacts ranging from increased maintenance costs for university administrations to increased pressures on academic performance of students and faculty members. The LSTC has performed more than 1 million tests that have helped mitigate SARS-CoV-2 transmission for nearly 2000 cases by informing individual quarantine measures and state contact tracing programs. High-complexity diagnostics, such as RTqPCR, maintain numerous components that have significant impact on the reproducibility of the assay and all potential pooling formats would require specific validation. An efficient workflow design at the LSTC involves aliquoting fresh samples into five 1-mL 96-well plates, which then are combined into a single master 1-mL 96-well plate for extraction. Sample tracking is facilitated by the laboratory information management system and impact to turnaround times is expected to amount to no more than an additional 24 hours, allowing the LSTC to keep reporting to fewer than 48 hours. Apparent volumes for routine population screening present a significant burden that a valid group testing strategy can help alleviate by providing an alternative that is both economically and environmentally beneficial.

\section{Acknowledgments}

We thank Dr. Christopher Bilder for accommodating our inquiries, Ciara Cheli-Colando for providing metrics, and the tireless efforts of all administrative and technical staff at the LSTC.

\section{Supplemental Data}

Supplemental material for this article can be found at http://doi.org/10.1016/j.jmoldx.2021.09.001.

\section{References}

1. Emanuel EJ, Persad G, Upshur R, Thome B, Parker M, Glickman A, Zhang C, Boyle C, Smith M, Phillips JP: Fair allocation of scarce medical resources in the time of Covid-19. N Engl J Med 2020, 382: 2049-2055

2. Paltiel AD, Zheng A, Walensky RP: Assessment of SARS-CoV-2 screening strategies to permit the safe reopening of college campuses in the United States. JAMA Netw Open 2020, 3:e2016818

3. Sharifi A, Khavarian-Garmsir AR: The COVID-19 pandemic: impacts on cities and major lessons for urban planning, design, and management. Sci Total Environ 2020, 749:142391

4. Jayaweera M, Perera H, Gunawardana B, Manatunge J: Transmission of COVID-19 virus by droplets and aerosols: a critical review on the unresolved dichotomy. Environ Res 2020, 188:109819

5. Moghadas SM, Fitzpatrick MC, Sah P, Pandey A, Shoukat A, Singer BH, Galvani AP: The implications of silent transmission for the control of COVID-19 outbreaks. Proc Natl Acad Sci U S A 2020, 117: 17513-17515 
6. Ward S, Lindsley A, Courter J, Assa'ad A: Clinical testing for COVID19. J Allergy Clin Immunol 2020, 146:23-34

7. Padgett LR, Kennington LA, Ahls CL, Samarasinghe DK, Tu YP, Wallander ML, Cooper SD, Elliott JS, Rains D: Polyester nasal swabs collected in a dry tube are a robust and inexpensive, minimal selfcollection kit for SARS-CoV-2 testing. PLoS One 2021, 16:e245423

8. Hitt BD: Group testing identification: objective functions, implementation, and multiplex assays (Doctoral dissertation). [Lincoln (NE)]: University of Nebraska. 2020, UNL Digital Commons Available at https://digitalcommons.unl.edu/dissertations/AAI27956346. Accessed February 2, 2021

9. Abdalhamid B, Bilder CR, Garrett JL, Iwen PC: Cost effectiveness of sample pooling to test for SARS-CoV-2. J Infect Dev Ctries 2020, 14:1136-1137

10. Yelin I, Aharony N, Tamar ES, Argoetti A, Messer E, Berenbaum D, Shafran E, Kuzli A, Gandali N, Shkedi O, Hashimshony T, MandelGutfreund Y, Halberthal M, Geffen Y, Szwarcwort-Cohen M,
Kishony R: Evaluation of COVID-19 RT-qPCR test in multi sample pools. Clin Infect Dis 2020, 71:2073-2078

11. Chong BSW, Tran T, Druce J, Ballard SA, Simpson JA, Catton M: Sample pooling is a viable strategy for SARS-CoV-2 detection in lowprevalence settings. Pathology 2020, 52:796-800

12. Chadha J, Khullar L, Mittal N: Facing the wrath of enigmatic mutations: a review on the emergence of SARS-CoV-2 variants amid COVID-19 pandemic. Environ Microbiol 2021, [Epub ahead of print] doi:10.1111/1462-2920.15687

13. Bal A, Destras G, Gaymard A, Stefic K, Marlet J, Eymieux S, Regue H, Semanas Q, d'Aubarede C, Billaud G, Laurent F, Gonzalez C, Mekki Y, Valette M, Bouscambert M, Gaudy-Graffin C, Lina B, Morfin F, Josset L: Two-step strategy for the identification of SARS-CoV-2 variant of concern 202012/01 and other variants with spike deletion H69-V70, France, August to December 2020. Euro Surveill 2021, 26:2100008 